\title{
Design of Miniaturized Ultra Wideband Microstrip Antenna Fed by Coplanar Waveguide
}

\author{
Wei DINGa ${ }^{a}$, , Dong-Sheng WU ${ }^{\mathrm{b}}$, Lan-Lan PING ${ }^{\mathrm{c}}$, Jing ZHANG ${ }^{\mathrm{d}}$, \\ Wen-Li LIANG \\ AnHui Jianzhu University, China \\ a1559404606@qq.com, b87764309@qq.com, '1831618767@qq.com, d1938340511@qq.com, \\ e1654992810@qq.com \\ *Corresponding author
}

Keywords: Ultra Wideband Antenna, Coplanar Waveguide, Miniaturization.

\begin{abstract}
The Ultra-Wideband antenna can effectively improve the system performance, such as range resolution, anti-jamming performance and complex target recognition. It is very important to study the UWB antenna. In this paper, the UWB antenna is discussed and studied, and the influence of antenna size miniaturization on the antenna bandwidth is discussed and the performance of the antenna is analyzed by the method of feeding and the shape of the antenna. The coplanar waveguide feed and the appropriate topology can effectively improve the bandwidth.
\end{abstract}

\section{Introduction}

The antenna can be divided into transmitting antenna and receiving antenna, which is an electronic device for transmitting and receiving electromagnetic wave signal. The transmitting antenna is the electromagnetic wave signal transmission equipment into radio signals and transmitted to the receiving antenna in free space, on the contrary, the radio signals received from free space into the electromagnetic wave signal[1-4].

UWB antenna[5-8] plays an important role in electronic countermeasure equipment, and it has been widely used in radar and other systems[9]. With the rapid development of high frequency integrated circuits, miniaturization system has become the trend of development. However, the miniaturized antenna will affect the other performance of the antenna, such as the bandwidth of the antenna, the gain of the antenna, introduced in the literature several UWB antennas are using coplanar waveguide feed mode, and combined with the structure of the wide gap, the combination of the two will introduce coupling between the patch and the gap, thereby increasing the characteristic of broadband antenna, which has been lack of the larger or smaller gain. Therefore, the main goal of the miniaturized antenna is to minimize the physical size of the antenna without reducing the other performance requirements of the antenna. Therefore, the UWB antenna has become a hot research topic in the field of antenna design[10].

\section{Designing Process}

In this paper, a compact ultra wideband monopole antenna is designed and fabricated. The shape of the radiating patch is similar to that of the sector, and the coplanar waveguide is used to realize the design of UWB antenna. Dielectric substrate of antenna is Rogers2000, through the basic theory of micro strip antenna, calculated the size of rectangular antenna patch and designed a miniaturized antenna. Use the way of coplanar waveguide to increase the bandwidth. Dielectric substrate of antenna is Rogers2000, dielectric constant is 2.55 . Dielectric substrate thickness is $0.722 \mathrm{~mm}$. The structure of the antenna is shown in Fig. 1, dielectric substrate size is $23 \mathrm{~mm} \times 20 \mathrm{~mm}$. The length and width of rectangular patch are calculated by the formula of rectangular patch micro strip antenna is $11.26 \mathrm{~mm} \times 9.8 \mathrm{~mm}$. Feeder length and width are $6.6 \mathrm{~mm} \times 2.2 \mathrm{~mm}$. Dimensions of the floor at both ends of the feeder is $5 \mathrm{~mm} \times 7.9 \mathrm{~mm}$. 


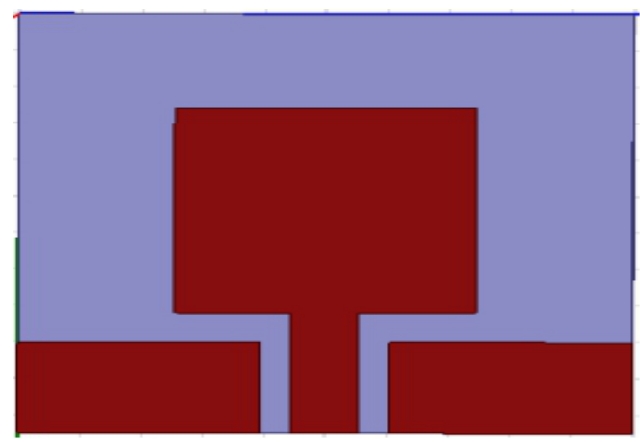

Fig. 1. Rectangular monopole antenna fed by coplanar waveguide

The radiation patch shape based on the size of the change. The radiation patch is cut into a narrow width of the isosceles trapezoid, the structure shown in Fig. 2. Half of the isosceles trapezoid on the bottom and the lower bottom difference, which cut off the bottom of the triangle, as in the variable $x_{1}$. The HFSS of this variable is simulated and optimized.

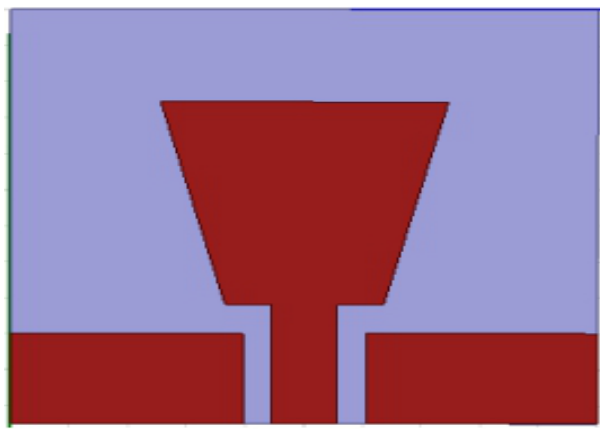

Fig. 2 Ladder monopole antenna structure

The bandwidth of the antenna can be further improved, in the bottom of the trapezoidal radiation patch adds an upper part of the ellipse, the upper bottom of the trapezoid is two times of the long axis of the ellipse, and the radiation patch shape and fan are similarity. Finally, get the antenna structure size as shown in Fig. 3, the size of the antenna such as a Table 1.

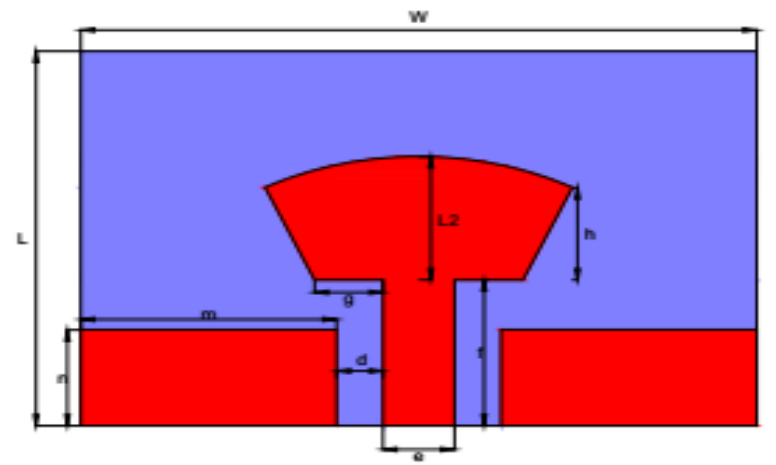

Fig. 3. Structure of ultra wideband monopole antenna

Table 1 the structure of ultra wideband monopole antenna

\begin{tabular}{|c|c|c|c|c|}
\hline $\mathrm{W} / \mathrm{mm}$ & $\mathrm{L} / \mathrm{mm}$ & $\mathrm{m} / \mathrm{mm}$ & $\mathrm{n} / \mathrm{mm}$ & $\mathrm{e} / \mathrm{mm}$ \\
\hline 23 & 20 & 8.3 & 5 & 2.2 \\
\hline $\mathrm{d} / \mathrm{mm}$ & $\mathrm{g} / \mathrm{mm}$ & $\mathrm{f} / \mathrm{mm}$ & $\mathrm{h} / \mathrm{mm}$ & $\mathrm{L} 2 / \mathrm{mm}$ \\
\hline 0.6 & 2.33 & 6.6 & 9.8 & 12.66 \\
\hline
\end{tabular}




\section{Result Analysis}

The micro strip antenna fed with coplanar waveguide, which has great influence on the performance gap between the ground floor and on both sides of the antenna feeder for the antenna. Therefore, the first of antenna and ground floor between the gaps on both sides of the D simulation optimization, get the return loss of S11 versus D, the result is shown in Fig. 4.

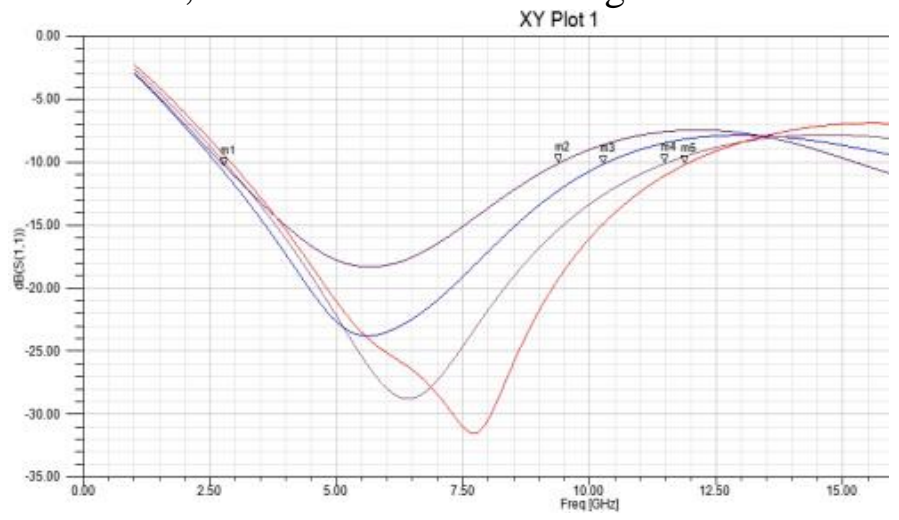

Fig. 4. Variation of the return loss curve

As can be seen from figure 4, the gap between the feeder and the ground floor to reduce the bandwidth of the antenna is also gradually increased. Take into account the production process of the final antenna, the gap should not be too small, which is easy to cause a short circuit, select the gap distance of $0.6 \mathrm{~mm}$ is more appropriate.

Fig. 2 shows the structure of the antenna is based on the rectangular Monopole Micro strip antenna is to be improved. In the other word, the rectangular radiation patch cut off the two triangles into, so that the radiation patch into an isosceles trapezoid.The variable $x_{1}$ set in HFSS, said the radiation was cut off the length of the triangle, and the structure of the antenna simulation optimization to improve the bandwidth of the antenna were compared to the rectangular antenna, the results are shown in Fig. 5.

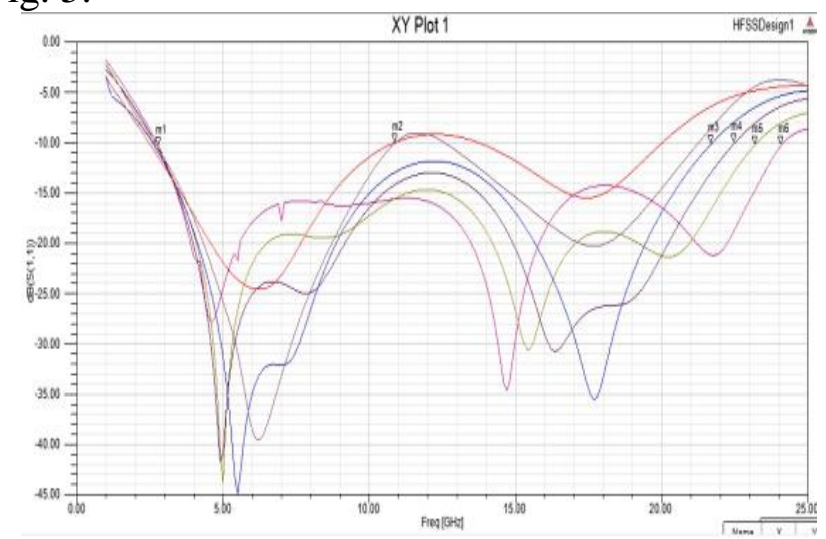

Fig. 5. Input return loss curve of the trapezoidal monopole UWB antenna

From the graph shown in figure 5 can see that, the bandwidth of the antenna is increasing gradually. Compare to the rectangular monopole antenna structure shown in Fig. 1 of the micro strip antenna bandwidth, more than doubled.

On the basis of the antenna structure shown in figure 2, on the top of the trapezoidal patch on the top of the patch with 1.5 ovals, the shape of the patch is similar to fan. In the HFSS simulation, observe the actual bandwidth of the antenna input return loss curve, as shown in Fig. 6.

With the increase of the semi elliptical patch, the antenna has a slight increase, the bandwidth is about $21 \mathrm{GHz}$. The optimized antenna not only improves the bandwidth greatly, but it as much two times as the rectangular micro strip of antenna and the physical size of the antenna is very small, so that the antenna miniaturization is achieved. 


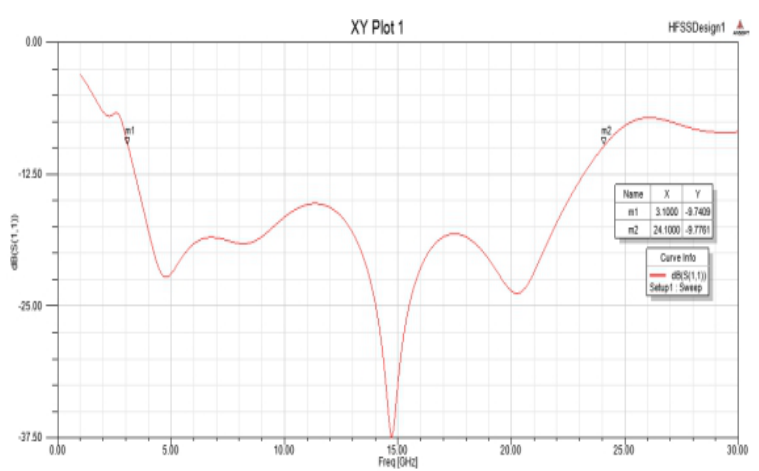

Fig. 6. Input return loss curve of sector monopole UWB antenna

The planar antenna pattern simulation results are shown in Fig. 7, the antenna E surface gain pattern is " 8 " shape, gain of one. The left antenna radiation efficiency is relatively low, increasing the bandwidth of the antenna so that the antenna radiation efficiency is reduced, so the antenna gain is also reduced.

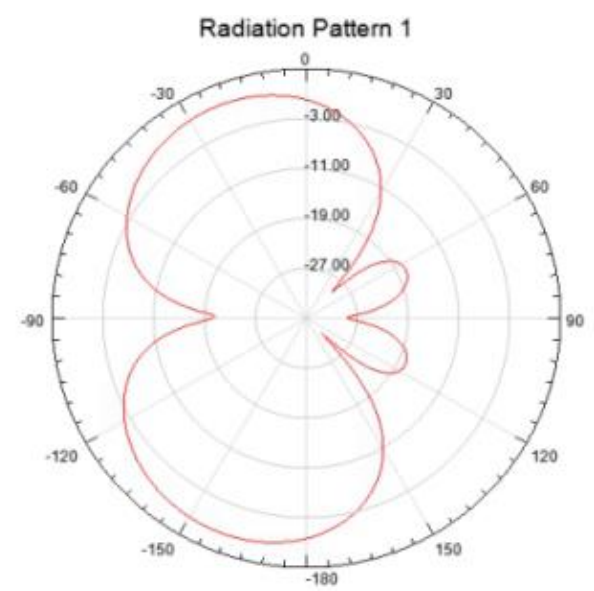

Fig. 7. E gain pattern of the improved monopole antenna

Sample processing and testing of this antenna, as it shown in Fig.8. The results of the measurement of the echo loss using the microwave network vector analyzer are shown in Fig. 9. It can be seen from the figure that the bandwidth of the antenna ranges from $2.5 \mathrm{GHz}$ to $25 \mathrm{GHz}$.

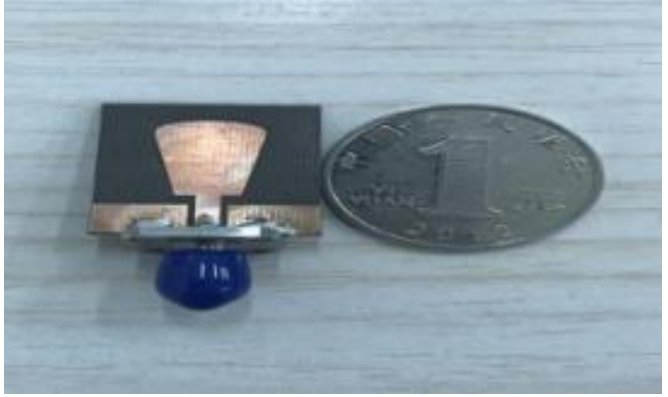

Fig.8. Sector monopole ultra wideband

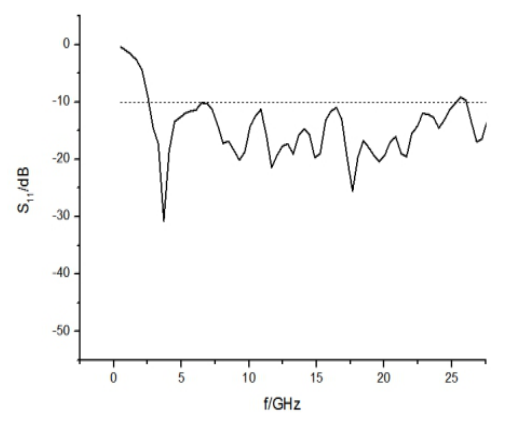

Fig. 9. Return loss curve of actual measurement antenna physical map

\section{Conclusion}

The miniaturization of the antenna is a special shape of the patch technology. In the rectangular patch on the basis to improve the shape of the radiation patch. Firstly, design a rectangular micro strip antenna with small size. In order to increase the bandwidth, the antenna is fed by coplanar waveguide. The simulation results show that the bandwidth of the antenna is only $8.7 \mathrm{GHz}$. In order to increase the bandwidth of the antenna, the rectangular patch is improved. Then based on the 
rectangular radiating patch, the rectangular patch cut two right triangles; into a narrow width of the isosceles trapezoid, optimized the bandwidth of the antenna is doubled.Finally, a semi elliptical radiation patch is added on the trapezoidal radiation patch, and the shape of the radiation patch looks like a fan, and the bandwidth of the antenna is increased slightly.

Finally, the bandwidth of the micro strip antenna is about $21 \mathrm{GHz}$, and the physical object is obtained according to the optimized size parameters, and the measured bandwidth is $22 \mathrm{GHz}$ with the microwave network analyzer.Miniaturized ultra wideband antenna is a very important topic. To achieve miniaturization of antenna technologies with its own advantages and disadvantages. Such as the radiation efficiency of dielectric substrate, high dielectric constant will reduce the size of the antenna,it will reduce the antenna and the bandwidth. Therefore, in the design of small antennas should be a good trade-off between bandwidth and gain requirements, select the most consistent with the design requirements of the program.

\section{Acknowledgment}

This work was supported by the National Natural Science Foundation of China (Grant No. 61471002).

\section{References}

[1] CHEN Xianming LIU Shuhuan,A novel kind of planar elliptical monopole ultra windband antenna[J].Piezoeletrics \& Acoustooptics,2016,38(1):162-165.

[2] Zhu Shouzheng, an an. Antenna theory and design [M]. Beijing: People's Posts and Telecommunications Press, 2009.

[3] Stutzman W L, Thiele G A. Antenna Theory and Design [M]. Hoboken, New Jersy: John Wiley \& Sons, Inc, 2012.

[4] R. Pandeeswari, S. Raghavan. Microstrip Antenna With Complementary Split Ring Resonator Loaded Ground Plane For Gain Enhancement [J]. Microwave And Optical Technology Letters, 2015,57(2):292-296.

[5]Chen Kenan, Wang Xin, ultra wideband UWB antenna design [J]. nuclear electronics and detection technology, 2009,29, (2):376-378.

[6]LI Tong, CAO Xiangyu, GAO Jun, et al. Design of highly band-notched ultra-wideband antenna [J]. Journal of Xidian University, 2016, 43(1): 41-46.

[7]Yang Xiao Dong, Rahman A., Abbasi Qammer H., et al.Electrically coupled tapered slot antenna with tunable notch[J].Microwave and Optical Technology Letters, 2011, 53(7) : 1558-1561.

[8]Abdollahvand M, Dadashzadeh G R, Ebrahimian H, Compact band-rejection printed monopole antenna for UWB application[J].IEICE Electronics Express,2011,8,(7) : 423-428.

[9] Chen Z N,Wu X H, Li H F, et al. Considerations for source pulses and antennas in UWB radio systems [J]. IEEE Transactions on Antennas and Propagation. 2004, 52(7):1739-1748.

[10] Liu Xianglong. Design and time domain characteristics analysis of small ultra wide band antenna [D]. doctoral dissertation of Xi'an Electronic and Science University. 